\title{
Inter-fibre failure of through-thickness reinforced laminates in combined transverse compression and shear load
}

\author{
Hao Cuia, b1 , António R. Melro ${ }^{\mathrm{c}}$, Mehdi Yasaee ${ }^{\mathrm{a}}$

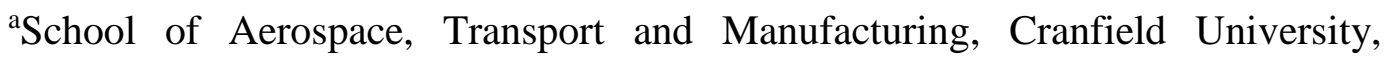 \\ Bedfordshire, UK \\ ${ }^{\mathrm{b}}$ Department of Engineering Science, University of Oxford, Oxford, UK \\ ${ }^{\mathrm{c} B r i s t o l}$ Composites Institute (ACCIS), University of Bristol, Bristol, UK
}

\begin{abstract}
Extensive studies have been reported on the improvement of through-thickness reinforcement to inter-laminar performance of composite laminates; current understanding on the in-plane performance is relatively limited, although it is also concerned in industrial application. The influence of through-thickness reinforcement (Z-pinning) on the inter-fibre failure in compression of unidirectional laminates was investigated. Both unpinned and Zpinned laminates were tested at four different off-axis angles, representing different combinations of transverse compression and in-plane shear stress. It was found that the stiffness of Z-pinned laminates decreased significantly in all off-axis angles. The failure strain and strength were reduced in shear dominated failure modes, while improved in the compression dominated failure modes by the presence of the Z-pins. A further investigation on the angle of failure plane was carried out and a comparison with analytical failure models is presented.

${ }^{1}$ Corresponding author: email: hao.cui@cranfield.ac.uk;

Phone: $+44(0) 1234754494$
\end{abstract}




\section{Keywords:}

Z-pin; inter-fibre failure; strength; fibre volume fracture

\section{Introduction}

The inter-laminar strength of carbon fibre reinforced composite laminates is generally much lower than that in the fibre direction. Accordingly, delamination and other inter-fibre failure modes in transverse direction have been a major concern in the development of composite structures $[1,2]$. Through-thickness reinforcement methods, such as Z-pinning, using metallic or carbon fibre rods inserted through the laminate thickness, have been studied in the last decades for its great potential in increasing damage tolerance of composite structures [3], namely in offering resistance to the growth of delamination.

Apparent fracture toughness in Mode I delamination of composite laminates was reported to be dramatically increased with Z-pins [4-9]. In mode II delamination, there is also a linear increase in apparent fracture toughness with the volume content of Z-pins [2, 8-10]. Z-pinning has also proven effective in decreasing fatigue crack growth rate [11]. The laminate through-thickness stiffness also exhibits significant increase with the insertion of Zpins [12]. The strength of composite joints may also be improved with Z-pinning, such as the work reported on T-joints and Adhesive joints [13-16].

The major benefit of Z-pinning to composite laminates is the improved out-of-plane performance, which has attracted most of the research on Z-pinned laminates [3]. The influence of Z-pinning on the in-plane properties has received much less attention. Existing work suggested that Z-pinning is detrimental to the in-plane stiffness and strength[3]; numerical simulation showed that the longitudinal modulus may drop by about $7-10 \%$ with Z-pinning of $2 \%$ volume fraction [12]. During Z-pin insertion process, fibres are pushed 
aside, creating a matrix rich zone around the pin and causing significant waviness of the fibres in the laminate [17]. This waviness may trigger crack initiation in the resin rich zone in longitudinal tension [18], as well as fibre kinking under longitudinal compression [18]. Experimental work has shown that longitudinal tensile strength is reduced by $27 \%$ while compressive strength drop is around 30\% due to Z-pinning [17]. The longitudinal modulus and strength were found to degrade linearly with the volume fraction of the Z-pins $[18,19]$. The flexural strength of laminates also decreases with Z-pinning [20].

Existing research on the in-plane properties of Z-pinned laminates has mainly focused on fibre dominated modes; few works have been reported on the matrix dominated inter-fibre failure behaviour. Off-axial compression tests of Z-pinned laminates were conducted by Steeves and Fleck [17], however, there is no comparison reported between off-axis strength of unpinned samples. Inter-fibre failure is one of the major failure modes in composite laminates due to the weak strengths of matrix and fibre-matrix interface. The fibre waviness [17], resin rich zone [19] and residual stress after cure [21], may have noticeable influence on the inter-fibre deformation and failure response. The in-plane performance is critical during sizing and analysis of composite structures. Inter-fibre stress may be dominating in some layers due to the fact that practical structures are mostly multi-directional. Many structures such as composite aero-engine fan blades may experience tension, torsion and bending load simultaneously, while delamination is also worth concerning due to the impact threat from foreign object. Therefore, a comprehensive understanding about the inter-fibre failure performance of Z-pinned laminates is vital for industrial applications.

As part of a systematic characterization work for understanding the Z-pinned laminates, an experimental investigation is reported for the first time here, to help understand the influence of Z-pinning on the inter-fibre failure strength of composite laminates, specifically here in the combined load of transverse compression and in-plane shear. 
Experimental set-up is introduced in Section 2, followed by a detailed investigation on the microstructures of Z-pinned laminates in Section 3. Experimental results are presented in Section 4, in which the influence of Z-pinning on the stiffness and strength of laminates is discussed.

\section{Material and methods}

\subsection{Manufacturing}

The IM7/8552 material system was chosen for the manufacture of the composite laminates. Two laminated plates with 40 plies of unidirectional prepregs were prepared with one being Z-pinned prior to curing. The cure process was carried out within an autoclave following the material supplier's (Hexcel, UK) recommended cure cycle. T300/BMI Z-pins of $0.28 \mathrm{~mm}$ diameter were inserted into the laminates' through-thickness direction in a $4 \times 9$ grid with spacing between Z-pins of $1.75 \mathrm{~mm}$ (equivalent to $2 \%$ areal density) as illustrated in Fig.1a. The mechanical properties of T300/BMI can be found in [22]. The same arrangement was then rotated to different angles with respect to the fibre direction. Four different angles $(\beta)$ were used: $30^{\circ}, 45^{\circ}, 60^{\circ}$, and $90^{\circ}$, which provided gradual transition of stress condition from in-plane shear dominated failure to the pure transverse compression case. A three-dimensional configuration of Z-pinned sample is presented in Fig.1b. Due to the challenge of insertion process, misalignment of Z-pins with respect to the thickness direction was noticed, and the misalignment angle is around $9^{\circ}$. Unpinned samples with equal geometry were also prepared to serve as benchmarks in the forthcoming comparative study.

Each sample was machined from the panel using a fine diamond saw. This provided very smooth, defect free surface on all the edges of the samples. The top and bottom surfaces 
of the Z-pinned samples were gently polished after curing to remove the pin bulges to create a nominally flat surface.

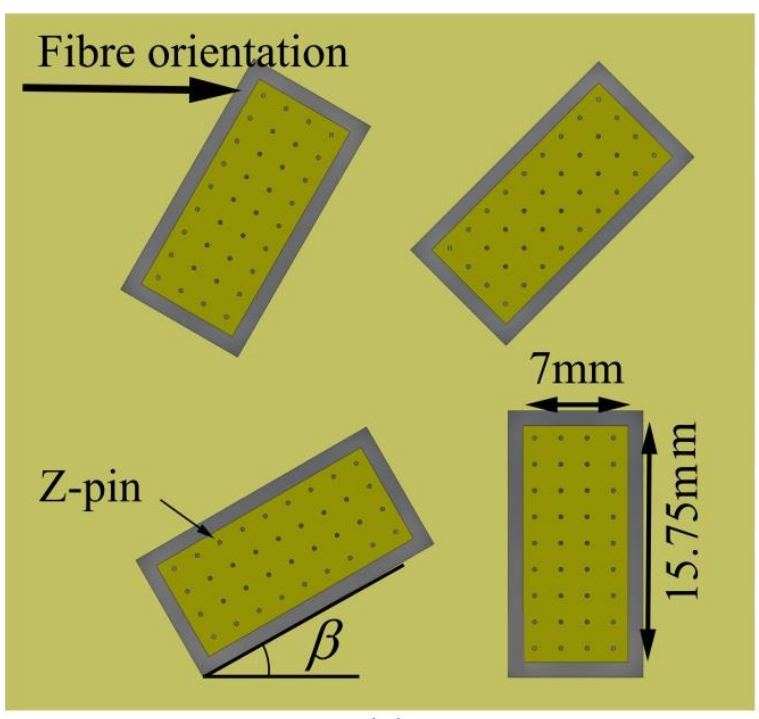

(a)

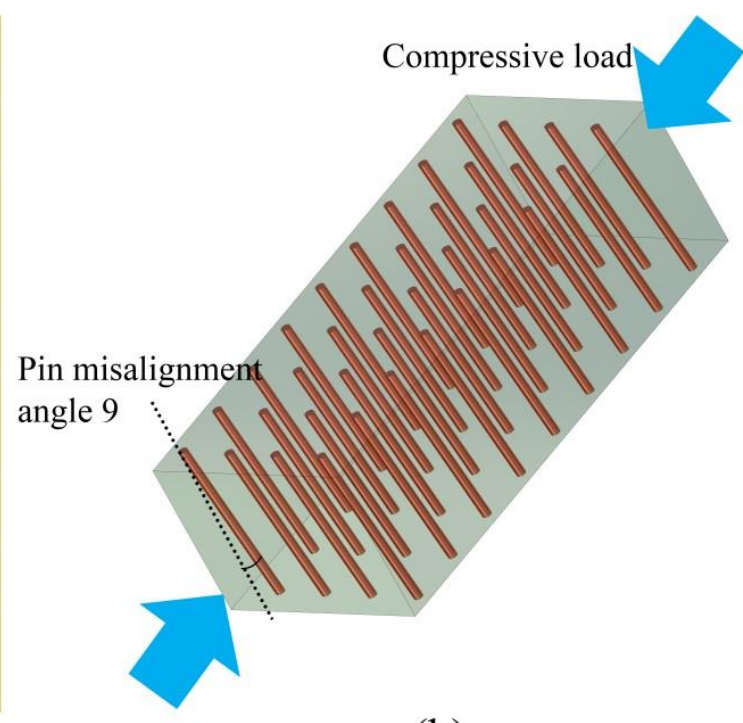

(b)

Fig.1 (a) preparation of Z-pinned laminates with Z-pins inserted in different patterns (b) three dimensional view of Z-pinned sample showing pin misalignment.

The cubic geometry was selected in this study, similar to the work by Koerber et al [20]. Frictional stress and stress concentration near the ends of samples hindered uniform stress distribution across the cross section. The non-homogeneity in stress distribution may results in premature failure, and the true material strength may be underestimated. The main purpose of this study is to evaluate the influence of Z-pinning on the inter-fibre failure response of unidirectional laminates. Considering the fact that both Z-pinned and unpinned samples share similar geometry, the non-homogeneity in stress distribution shouldn't affect comparative study undertaken here. The interface between the sample and the fixture was lubricated before each test, to minimize the effect of friction. 


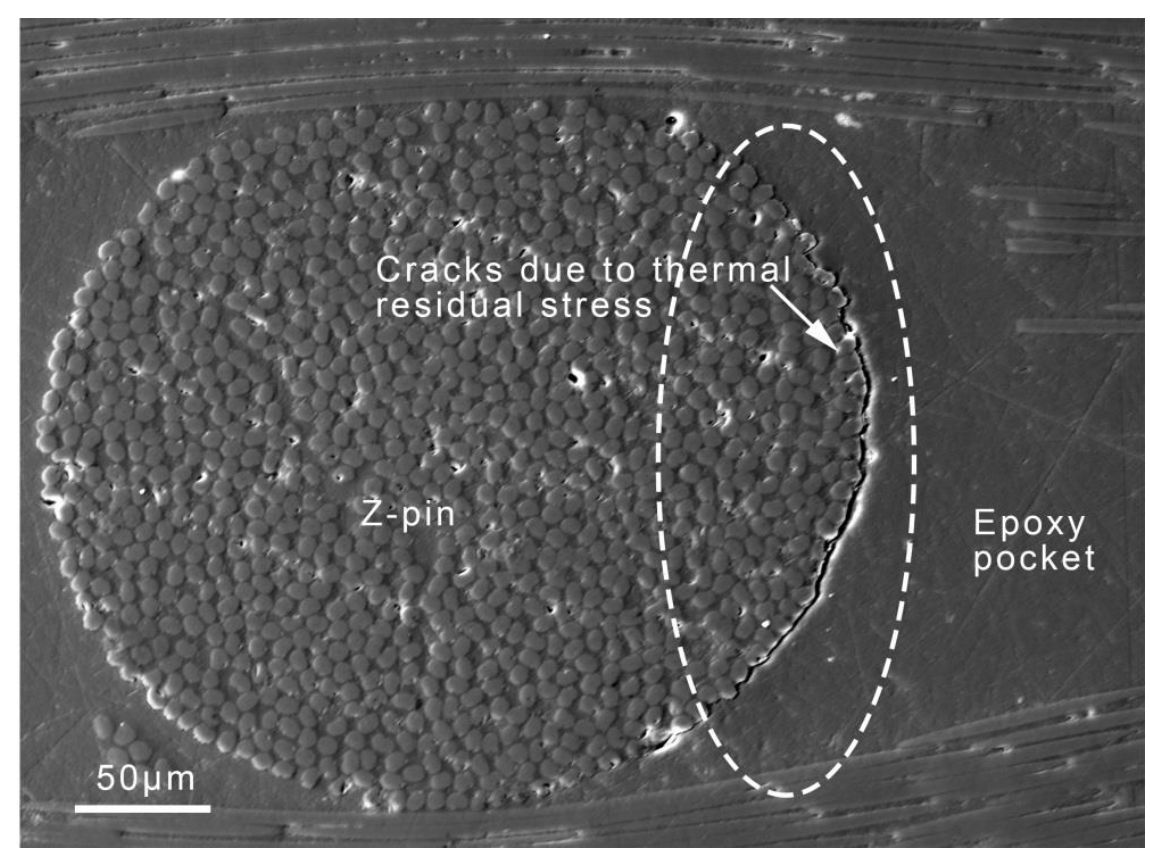

Fig.2. Debonding around Z-pin after curing caused by residual thermal stress

The Z-pinning process resulted in increased defects in the composite laminates. Residual thermal stresses after curing have resulted in partial debonding of Z-pin from surrounding epoxy resin. As show in Fig.2, the debonded interface is certainly detrimental to the in-plane strength, which may grow at relatively low stress level due to the stress concentration at crack tip. Besides, the fibre waviness is significant around Z-pins as will be shown in Fig.4.

\subsection{Test set-up}

All tests were carried out on a standard Zwick Roell 250 test machine, at a constant displacement rate of $0.01 \mathrm{~mm} / \mathrm{s}$. The samples were loaded via flat-end steel fixture as illustrated in Fig.3. The ends of the loading fixture ensured to be perpendicular to the loading axis. Laser extensometer was used to track the relative displacement between both ends of the sample. The failure process was recorded with a camera at a frame rate of 2 frames per second. Each sample was painted with a speckle pattern on white background. A digital image correlation (DIC) method was then employed to capture the strain field on the specimen surface, using the GOM ARAMIS ${ }^{\circledR}$ software. The in-plane strain field for samples 
of off-axis angle $30^{\circ}$ and $45^{\circ}$ was analysed with DIC methods to get the normal and in-plane shear strains in the local material coordinate system. These two off-axis angles were chosen because of the shear dominated nonlinear deformation in these loading cases [23].

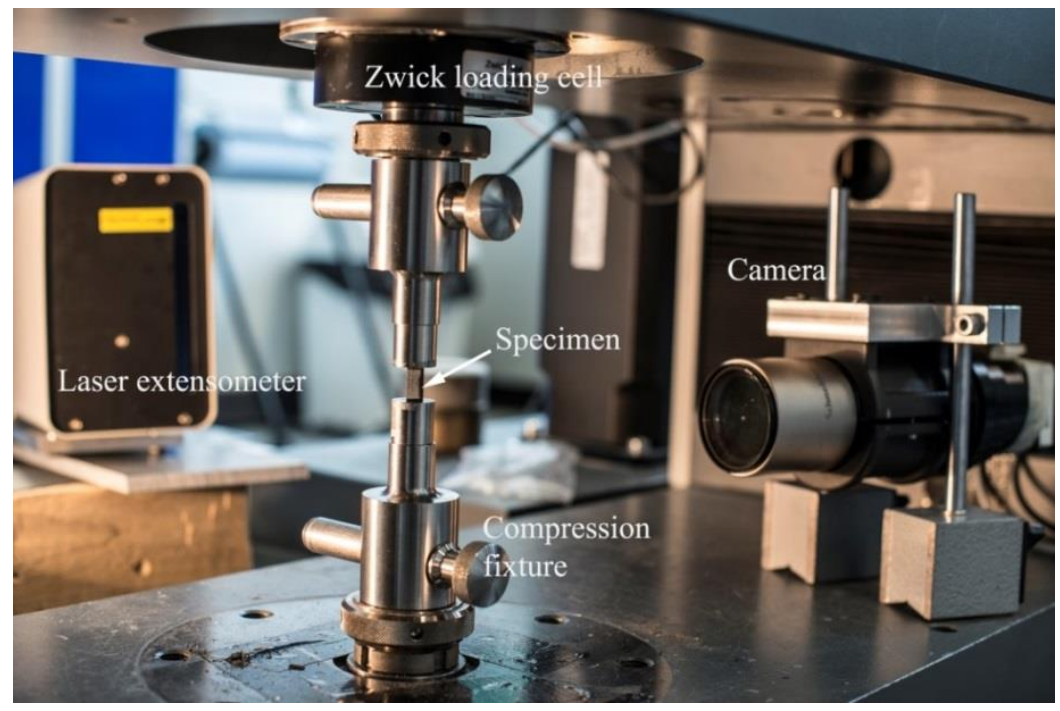

Fig.3 The compression experiment configuration.

\subsection{Data process}

The true axial strain $\varepsilon_{\mathrm{xx}}$ is determined with the measured displacement as:

$$
\varepsilon_{x x}=\ln \left(\frac{l-\delta}{l}\right)
$$

where $l$ is the length of the sample, and the $\delta$ is the relative displacement at the boundary of the sample measured with laser extensometer. The true axial stress is calculated:

$$
\sigma_{x x}=\frac{F}{A} \frac{l-\delta}{l}
$$

in which $F$ is the measured force and $A$ is the cross-section area. The apparent stress components in off-axis compression tests were determined as [23]: 


$$
\begin{aligned}
& \sigma_{22}=\sigma_{x x} \sin ^{2} \beta \\
& \tau_{12}=-\sigma_{x x} \sin \beta \cos \beta
\end{aligned}
$$

where $\sigma_{22}$ is the normal stress, and $\tau_{12}$ is the in-plane shear stress in local material coordinate system.

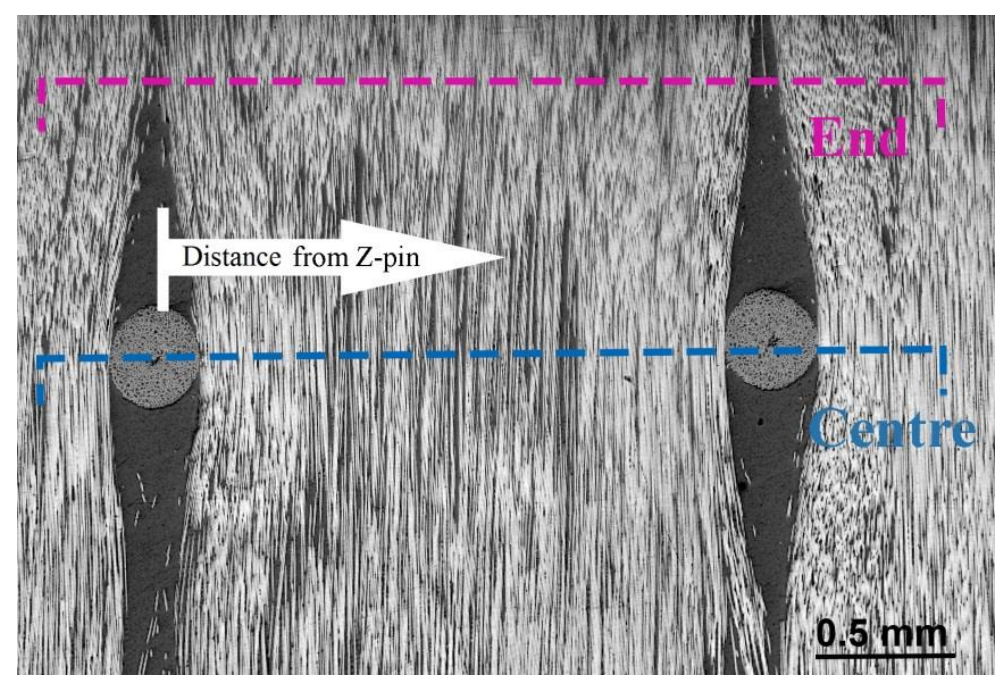

Fig.4. the resin pocket and fibre waviness in Z-pinned laminates

\section{Microstructural characterisation}

One of the outcomes of inserting Z-pins in a fibre reinforced composite is the formation of fibre waviness, and a resin rich zone around the Z-pins as shown in Fig.4. In order to investigate the fibre volume variation in Z-pinned laminates and characterise the area of influence of an individual pin, two cross-sections were defined and analysed.

The first cross-section crosses two neighbouring pins (identified as 'Centre' in Fig.4) while the second cross-section cuts the material in a more remote region ('End' in Fig.4). A high-resolution mosaic was created from smaller optical images and the result is shown in Fig.5a for the 'Centre' cross-section and in Fig.5b for the 'End' cross-section. The evolution of the fibre volume fraction within the area highlighted in Fig.5 was then measured using an open source image processing software ImageJ and the results are presented in Fig.6. The 
fibre distribution is very dense near the resin-rich area because the Z-pins have pushed fibres of the laminate aside and compressed them during insertion process, leading to considerable waviness in these fibres (see Fig.4). The averaged fibre volume fraction excluding resin-rich zone (see Fig.6) is very close to that of unpinned laminates. Including the volume of resinrich zone, the averaged fibre volume was about 0.56 at the 'Centre' section shown in Fig.4 (Z-pin fibres were not considered in the fibre volume), and 0.57 in the 'End' section. The unpinned samples were analysed as well, and its averaged fibre volume fraction was found to be between $0.63 \pm 0.03$. The Z-pins may have influence on the compaction of fibres during the curing process, and are responsible for the lower fibre volume fraction.
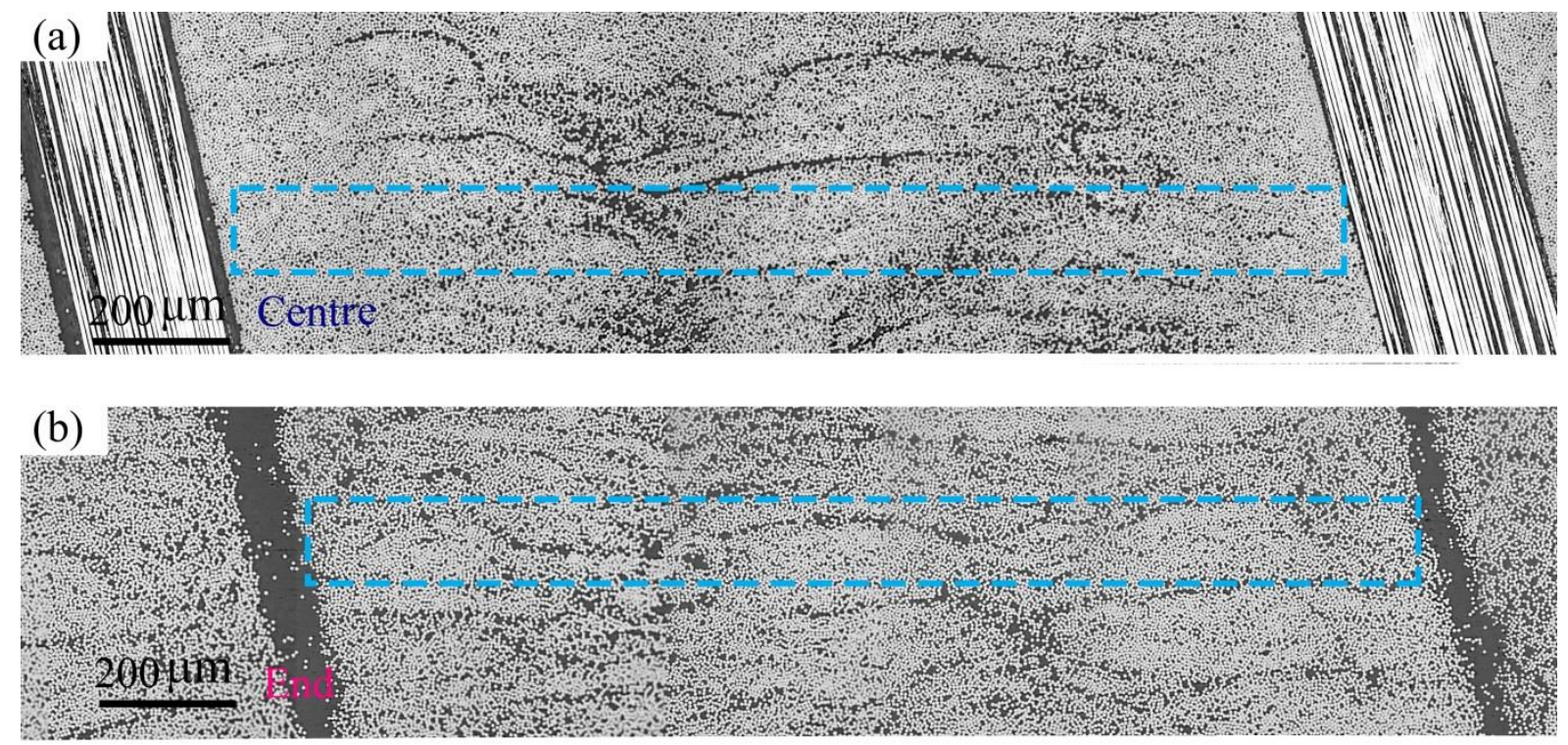

Fig.5. Micro-structure of Z-pinned laminates through the thickness direction: the crosssection at the (a) centre and (b) end of resin rich zone 


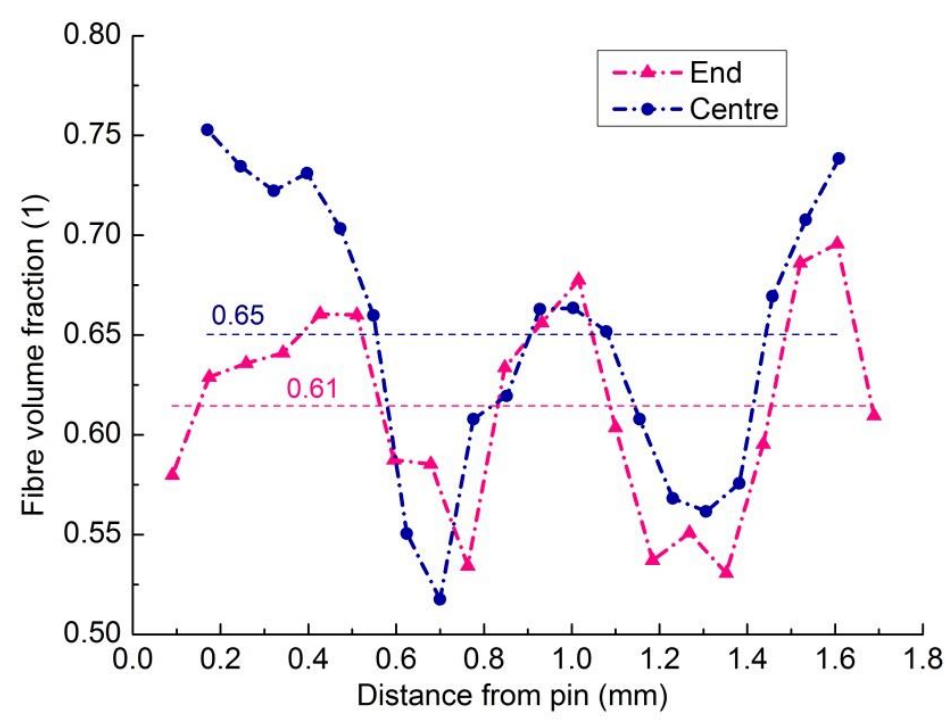

Fig.6 Fibre volume fraction of Z-pinned laminates

\section{Experimental results}

\subsection{Observation of failure types}

Three unpinned and four Z-pinned samples were tested for each off-axis angle, and a representative failed specimen for each test case is shown in Fig.7. All specimens failed along a fracture plane parallel to the fibre direction. For an off-axis angle of $\beta=30^{\circ}$, the fracture plane was following the insertion direction of Z-pins. Increasing the off-axis angle leads to an increase of the fracture angle relative to the thickness direction of the laminate. For both unpinned and Z-pinned samples in purely transverse compression tests $\left(\beta=90^{\circ}\right)$, the failure plane initiated at an angle around $50 \pm 5^{\circ}$ degrees in the plane normal to fibre direction, with respect to the normal direction of the laminate. Similar failure modes have also been reported for unpinned unidirectional laminates in [23]. At the macroscopic level, it can be concluded that there is no noticeable difference in the failure mode between unpinned and pinned laminates. 


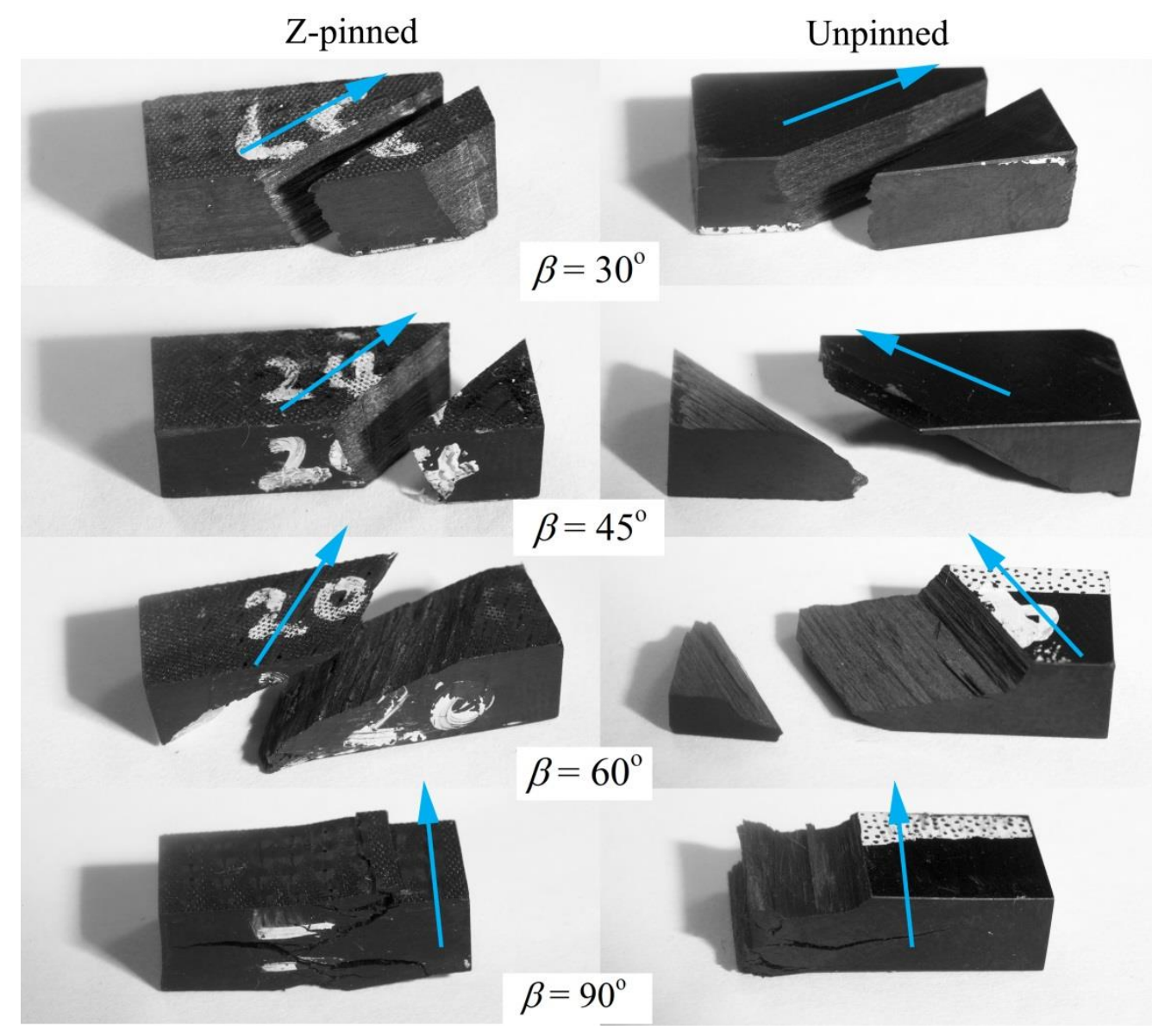

Fig.7 Failure modes for unpinned and Z-pinned samples with different off-axis angles.

\subsection{Stress-strain curves}

Representative stress-strain curves in the loading axis direction from all tests are plotted in Fig.8, where the strain was estimated using $\mathrm{Eq}(2)$ with the measurement from laser extensometer. Stress first evolved linearly with strain until nonlinearity emerged. The initiation of nonlinearity increased with the increase of off-axis angle, because the yield strength can be enhanced by transverse compressive stress [24]. The axial failure strain was between $3 \%$ and $7 \%$ depending on off-axis angle, detailed discussion on it will be provided in Section 4.3. 


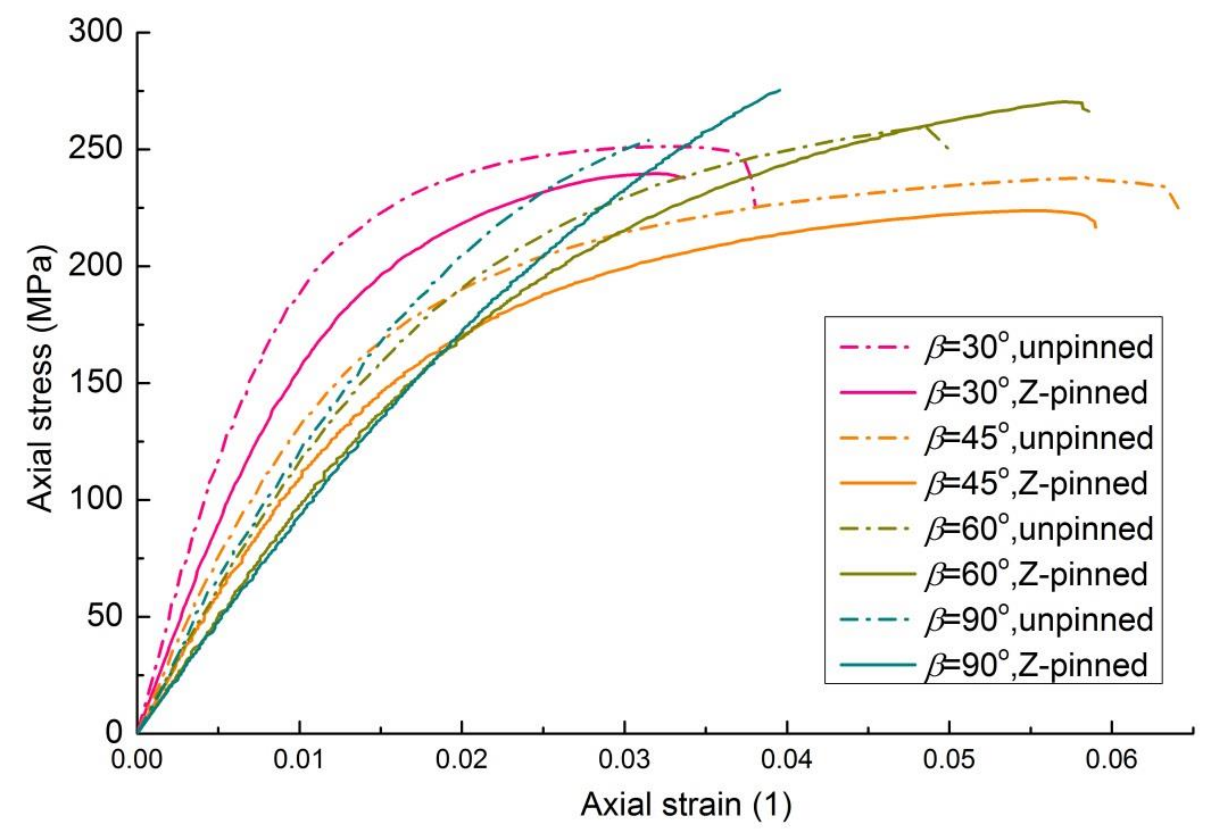

Fig. 8 The axial stress-strain curves along the loading direction.

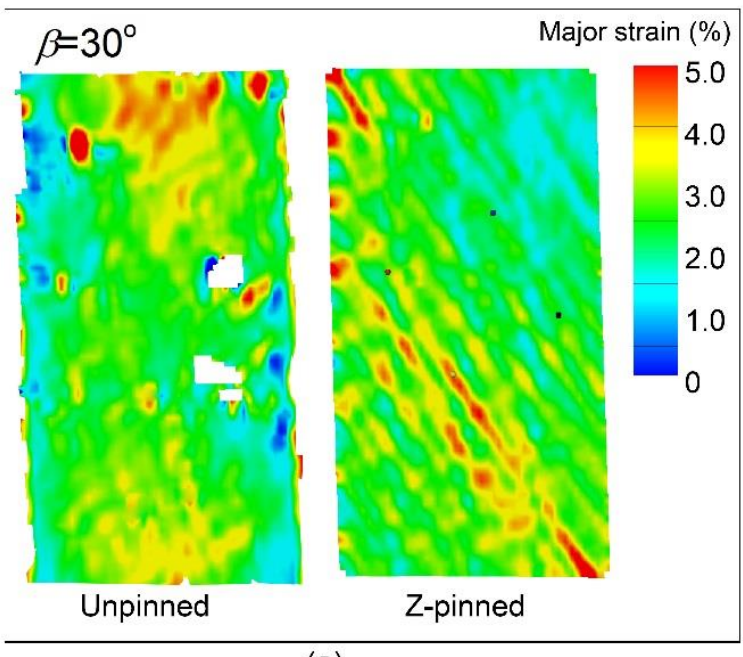

(a)

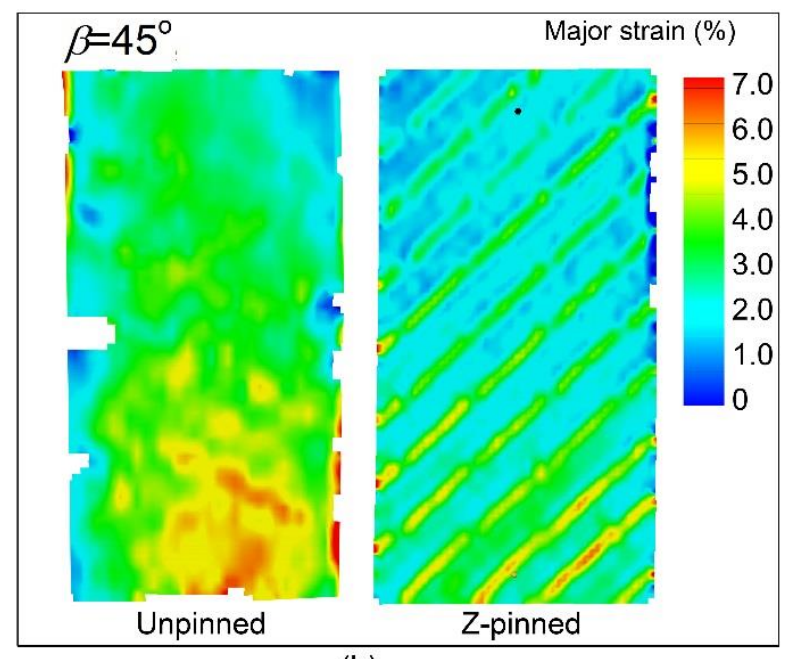

(b)

Fig.9 (a) major strain field at the axial strain of 0.032 for off-axis angle of $30^{\circ}$; (b) major strain field at the axial strain of 0.045 for off-axis angle of $45^{\circ}$;

The major strain on surface of samples were obtained from images taken by the USB camera, with the GOM Aramis DIC software, as shown in Fig.9 for tests at off-axis angle of $30^{\circ}$ and $45^{\circ}$. Due to the fact that the magnitude of strain is dependent on many parameters such as the selected facet size during DIC process, this figure should only be for indicting a 
global strain distribution, rather than any specific strain value. There is some high strain zoon near the boundary for unpinned samples, suggesting the influence of selected fixture on the experimental results, as discussed in section 2.1. The Z-pins have much more significant influence on the strain distribution than the boundary constraints introduced via loading interface. There is noticeable strain concentration following the orientation of resin pocket ( see Fig.4) in Z-pinned samples.

With the deformation field analysed using DIC methods, it is possible to calculate the transverse compression and in-plane shear response in local material coordinate system. The local stress components were calculated using $\mathrm{Eq}(3)$, to assemble the stress-strain response for the tests at off-axis angle of $30^{\circ}$ and $45^{\circ}$ presented in Fig. 10. It becomes evident that the nonlinearity is mainly attributed to the in-plane shear deformation component, for both unpinned and Z-pinned samples (see Fig.10). The unpinned samples exhibited considerable nonlinear compressive deformation when approaching the failure strength, while this nonlinearity seemed to be limited by the presence of the Z-pins. As shown in Fig.10b, the normal failure strain of the Z-pinned samples is noticeably lower than that of unpinned ones, which could be caused by existing debonding at pin-matrix interface (Fig.2). The localization of strain around Z-pins (shown in Fig.9) is also responsible for the reduced ductility of Zpinned laminates.

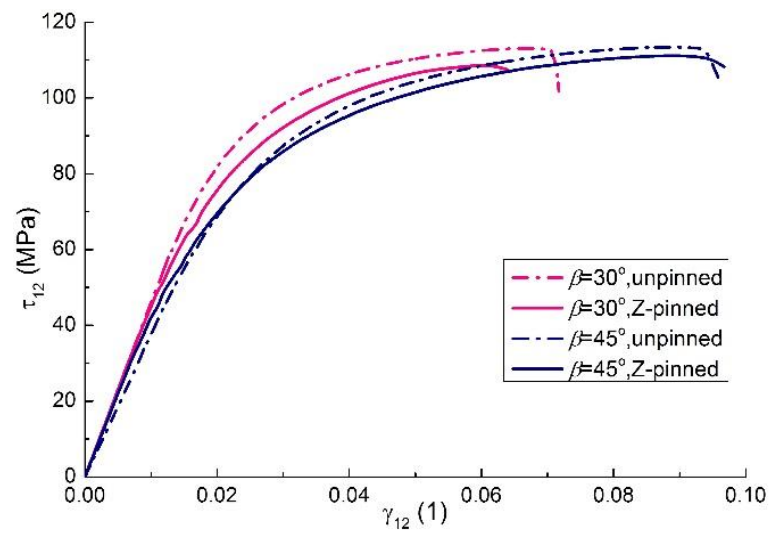

(a)

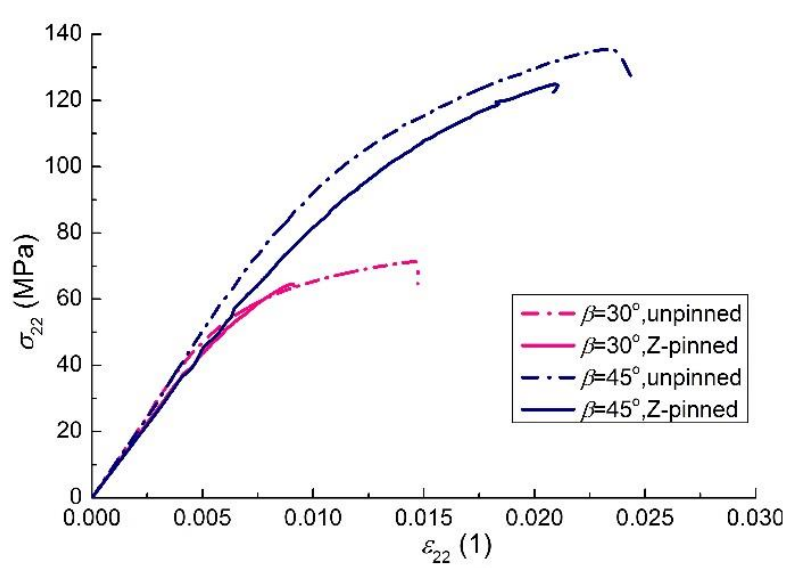

(b) 
Fig.10 Comparison of the in-plane deformation in material coordinate system for

$$
\beta=30^{\circ} \text { and } \beta=45^{\circ} \text {. }
$$

\subsection{Stiffness, failure strain and strength}

The elastic modulus of the laminates for each off-axis angle $\beta$ was investigated using the linear portion of the stress-strain curves of Fig.8 and results are presented in Fig.11a. The laminate modulus was reduced by Z-pinning in all investigated angles. This reduction in stiffness is justified by the waviness in the laminate plies caused by pin insertion [3], and by the presence of resin rich zones around each pin and the corresponding decrease in fibre volume fraction.

The critical stress at the moment of unstable failure surface propagation, from both unpinned and Z-pinned samples, is summarized in Fig.11b. Despite considerable scatters in all tests, it is observed that Z-pinning decreases the in-plane shear dominated fracture, as illustrated in the experiments with off-axis angles of $30^{\circ}$ and $45^{\circ}$. Similar results have been reported in tension tests of $\pm 45^{\circ}$ laminates [25]. The failure strength for off-axis angle of $60^{\circ}$ was not reduced by the presence of Z-pins, although the scatter for both tests is considerable. However, it is interesting to notice that the purely transverse compression strength was improved by Z-pinning. The influence of Z-pinning on the inter-fibre failure strength shows clear dependence on the stress conditions. 


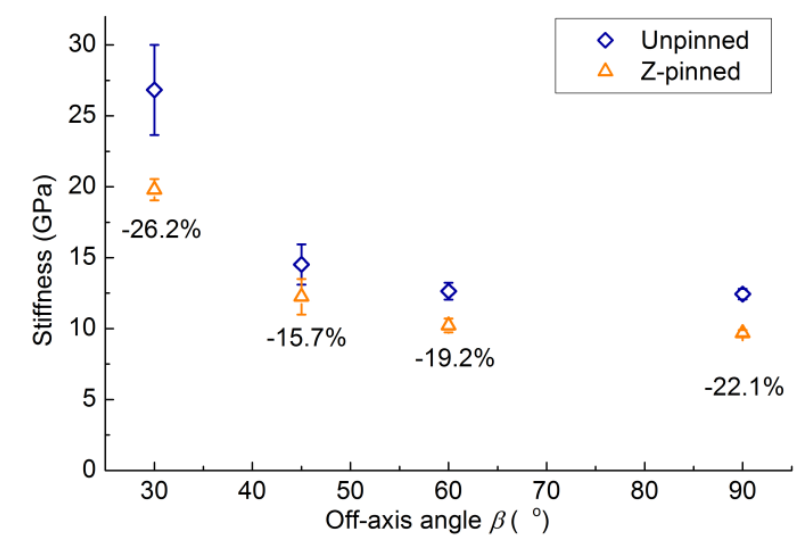

(a)

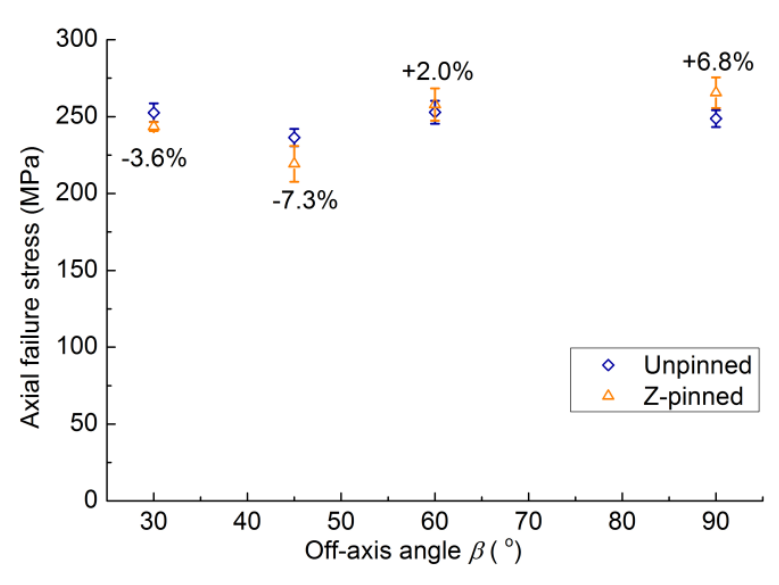

(b)

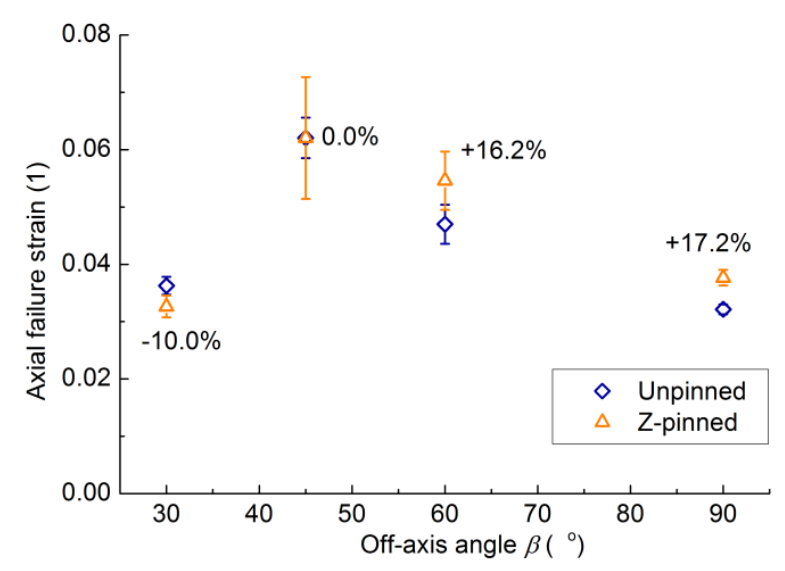

(c)

Fig.11 Influence of Z-pinning on the (a) stiffness, (b) strength and (c) failure strain for different off-axis loading angles.

The strain at final failure for both unpinned and Z-pinned laminates is shown in Fig.11c. When the failure is dominated with in-plane shear stress $\left(\beta=30^{\circ}\right)$, the failure strain is reduced by about $10 \%$ by Z-pin presence. The failure strain is not influenced by Z-pinning for an off-axis angle of $45^{\circ}$. However, larger intervals of results were observed in the Zpinned samples. For the samples loaded predominantly with transverse compression stress, Zpinning leads to an increase in the failure strain. The purely transverse compressive failure strain increases by approximately $17 \%$, for the Z-pin aerial density of $2 \%$ used in this study. This increase may be attributed to two factors: (1) increase in the failure stress with the presence of the Z-pins and (2) the decreased stiffness modulus leading to greater strain 
required to reach the equivalent stress level in unpinned laminates. The influence of $\mathrm{Z}$ pinning on in-plane failure transitioned from negative to position at the angle between $30^{\circ}$ and $60^{\circ}$. Considering the misalignment angle illustrated in Fig.1, the local Z-pin angle deviation may be responsible for the relatively large scatter in $45^{\circ}$ experiments.

There were 3-5 samples tested for each angle for both unpinned and Z-pinned samples, and the actual scatter and error in Fig.11 should be higher if more samples were tested. Although only limited number of samples were tested in this work, the influence of Z-pins on the in-plane performance is evident at least from a qualitative manner, which may be further confirmed with more samples.

\subsection{Micromechanical failure mechanisms}
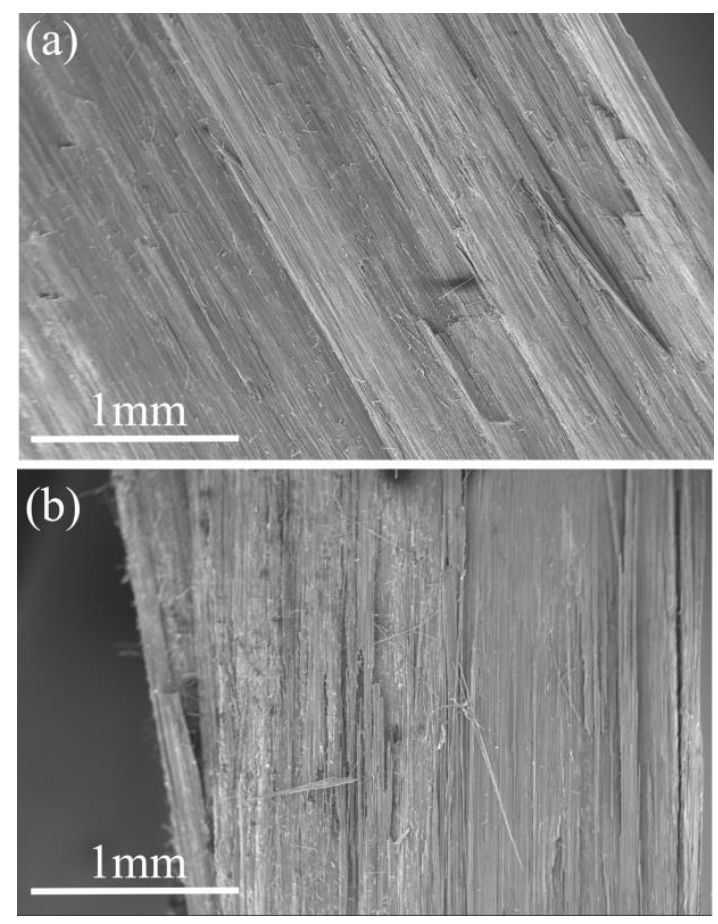

Fig. 12 Failure surface of unpinned samples (a) $\beta=60^{\circ}$; (b) $\beta=90^{\circ}$.

The failure processes were predominantly unstable during tests, making it very difficult to specify the location for damage initiation on the fracture surface. However, the fracture surface was reasonably consistent for each test case, and a representative surface was 
selected to study the failure mechanisms at microscale with SEM imaging. All unpinned samples exhibited very similar fracture surface morphology for each magnification scale with the failure surface of samples with $\beta=60^{\circ}$ and $\beta=90^{\circ}$ shown in Fig. 12 for reference. Interfibre fracture dominated the failure process, while few fibre ruptures were observed in the $90^{\circ}$ test cases.
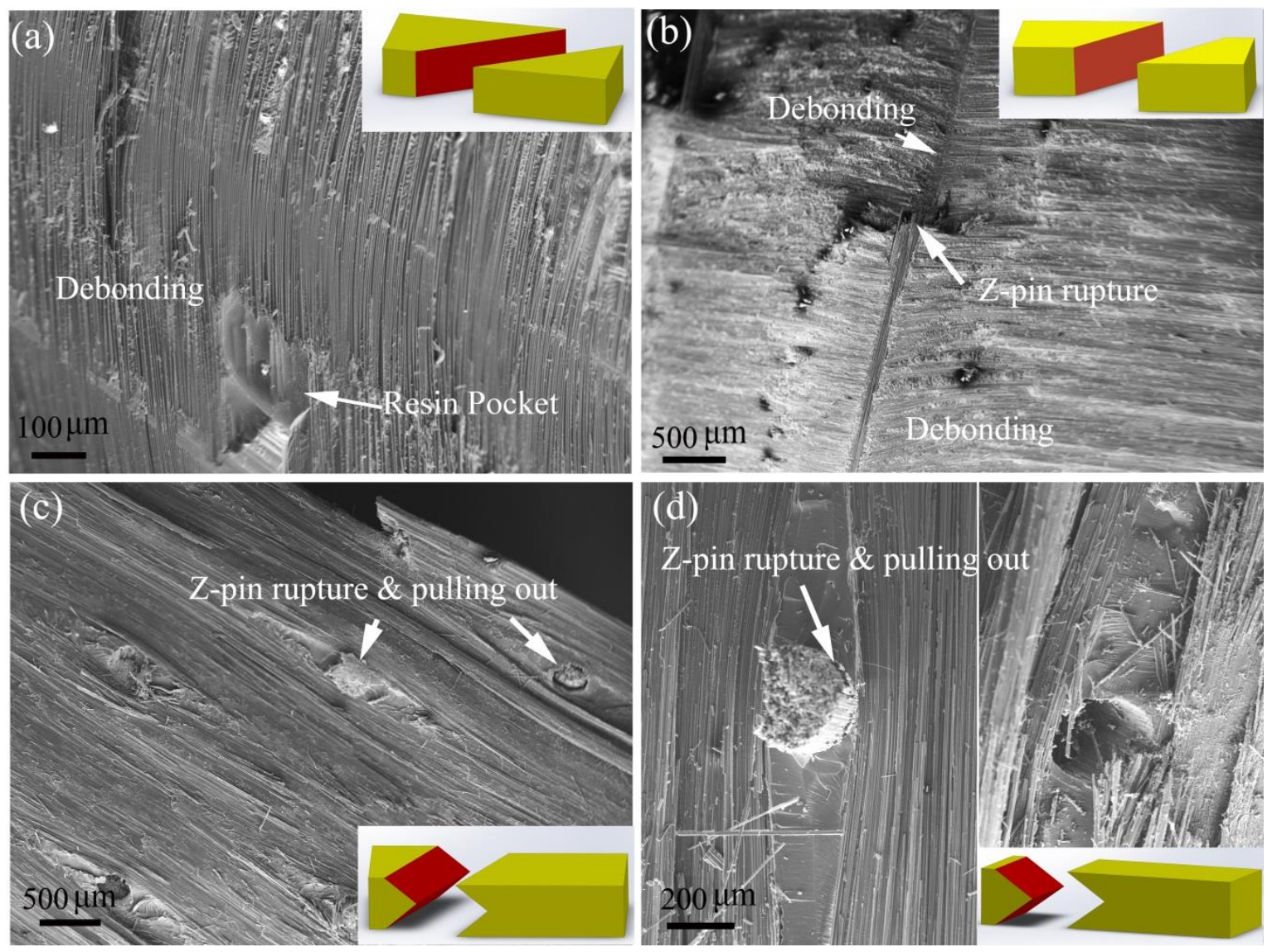

Fig.13 Failure mechanisms of Z-pinned laminates in compression tests of different off-axis

$$
\text { angles: (a) } \beta=30^{\circ} \text {; (b) } \beta=45^{\circ} \text {; (c) } \beta=60^{\circ} \text {; (d) } \beta=90^{\circ} \text {. }
$$

The fracture surfaces of Z-pinned samples are shown in Fig.13. The fracture planes on these samples are illustrated with red surface in the blocks. At an angle of $\beta=30^{\circ}$, failure occurred between the resin rich zone and the surrounding fibres, with a few resin pockets visible in Fig.13a. As shown in Fig.4\&6, there is a very high fibre volume fraction next to the Z-pins, causing a relatively smooth adhesion interface between the laminate and resin-rich 
zone. The debonding between Z-pin and epoxy (Fig.2) may be largely responsible for stimulating the failure process.

The failure mechanisms started to change when the off-axis angle increases from $30^{\circ}$ to $45^{\circ}$. As shown in Fig.13b, interfacial failure between resin rich zone and fibres can be observed, which may be responsible for the decrease in failure strength. However, this interfacial crack didn't propagate through the entire laminate thickness, and migrated to the opposite side of the resin rich zone, shearing off the Z-pin during this process. As a consequence of the combined failure modes, the failure strain of Z-pinned laminates was similar to that of unpinned ones.

The crack path does not follow the resin rich interface for laminates with off-axis angle of $60^{\circ}$, as shown in Fig. $13 \mathrm{c}$. It propagates across the resin rich zone, causing all Z-pins near the failure surface to be sheared-off. Some Z-pins have failed with a small pultruded length from the failure surface meaning some pull-out has occurred during failure surface propagation in the laminates [26].

For an off-axis angle of $90^{\circ}$ (pure transverse compression), the Z-pins failed in a very similar way as that in the samples of $60^{\circ}$, as shown in Fig.13d. However, the pull-out phenomenon is more significant. The existence of Z-pin rupture and pull-out for off-axis angles of $60^{\circ}$ and $90^{\circ}$ is the main justification for the increase in strength observed in Fig.9b for these two off-axis loading angles. 


\section{Discussion on the transition of Z-pinning influence}

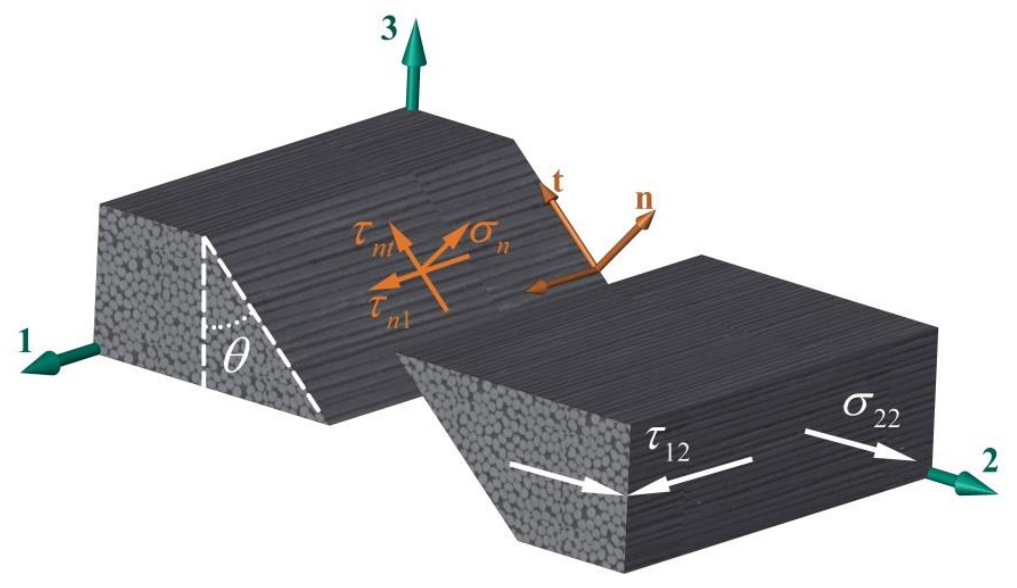

Fig.14 Illustration of the inter-fibre failure plane

The influence of Z-pinning on the failure strength varied from negative to positive as the change in off-axis angle. Such transition showed also clear correlation with the Z-pin failure modes introduced in the previous section. In this section, the authors try to explain the change of Z-pin failure mode, and therefore understand the transition of Z-pinning influence.

The failure modes of Z-pins is largely dominated by the global failure process of the laminates, due to the small Z-pin aerial density. The inter-fibre failure happens on a plane parallel to the fibres according to Puck's theory [23, 27, 28], as shown in Fig.14. The plane may be inclined by an angle $\theta$; only the two shear tractions $\tau_{\mathrm{n} 1}$ and $\tau_{\mathrm{nt}}$, and the normal stress $\sigma_{\mathrm{n}}$ contribute to failure. The quadratic form of Puck's criteria reads:

$e=\left(\frac{\tau_{\mathrm{n} 1}}{R_{\mathrm{n} 1}-p_{n 1} \sigma_{n}}\right)^{2}+\left(\frac{\tau_{\mathrm{nt}}}{R_{\mathrm{nt}}-p_{n t} \sigma_{n}}\right)^{2}=1$, for $\sigma_{22}<0$

where $e$ is the damage exposure, $p_{n 1}$ and $p_{n t}$ are the friction coefficient to account for the enhancement in shear strength due to compressive normal stress. $R_{\mathrm{n} 1}$ is the longitudinal inplane shear strength, which can be determined experimentally [29], and $R_{\mathrm{nt}}$ is the transverse shear resistance on the Puck failure plane and can be estimated by [30]: 
$R_{\mathrm{nt}}=\frac{Y_{C}}{2 \tan \theta_{f}}$

The friction coefficients can also be determined as [30]:

$p_{n t}=-\frac{1}{\tan 2 \theta_{f}}$

$p_{n 1}=p_{n t} \frac{R_{n 1}}{R_{n t}}$

in which $\theta_{f}$ is the failure plane angle under transverse compression, typically around $53^{\circ}$. The stress components on the failure plane can be calculated by rotating the stress $\sigma_{22}$ and $\tau_{12}$ with angle $\theta$. The angle of this failure plane $\theta$ is dependent on the stress state, and it can be determined by finding the angle that maximizes the damage exposure $e$ [28].

The failure angle $\theta$ can be determined for different combination of transverse compression and shear components, which can be calculated for different off-axis angle $\beta$ using $\mathrm{Eq}(3)$. The variation of inter-fibre failure angle $\theta$ with respect to off-axis angle $\beta$ is shown in Fig.15. When the off-axis angle is small, the sample is loaded mainly in shear, and the failure plane is aligned with the thickness direction. With the increase in off-axis angle, the failure plane rotates around the fibre axis in the laminate.

The response of Z-pins was very much dependent on the way they were loaded [26, 31]. In shear dominated failure, the resin-rich zone and the interface between resin pocket and surrounding laminate fibres weaken the intra-laminar strength (Fig.13 a), resulting in a failure plane parallel to the insertion direction of Z-pins. With increasing transverse compressive stress, the failure plane crosses through the Z-pin, resulting in initial debond and pull-out of the Z-pin, up to its complete failure in shear (Fig.13 c\&d). These processes consume considerable energy providing an additional bridging force and increasing the damage resistance. 
In summary, the imperfections caused by Z-pinning, such as a resin-rich zone, are responsible for the decrease in strength in shear-dominated failure modes while the bridging force brought by the Z-pins at the failure plane helps improve the strength under compression dominated loading conditions.

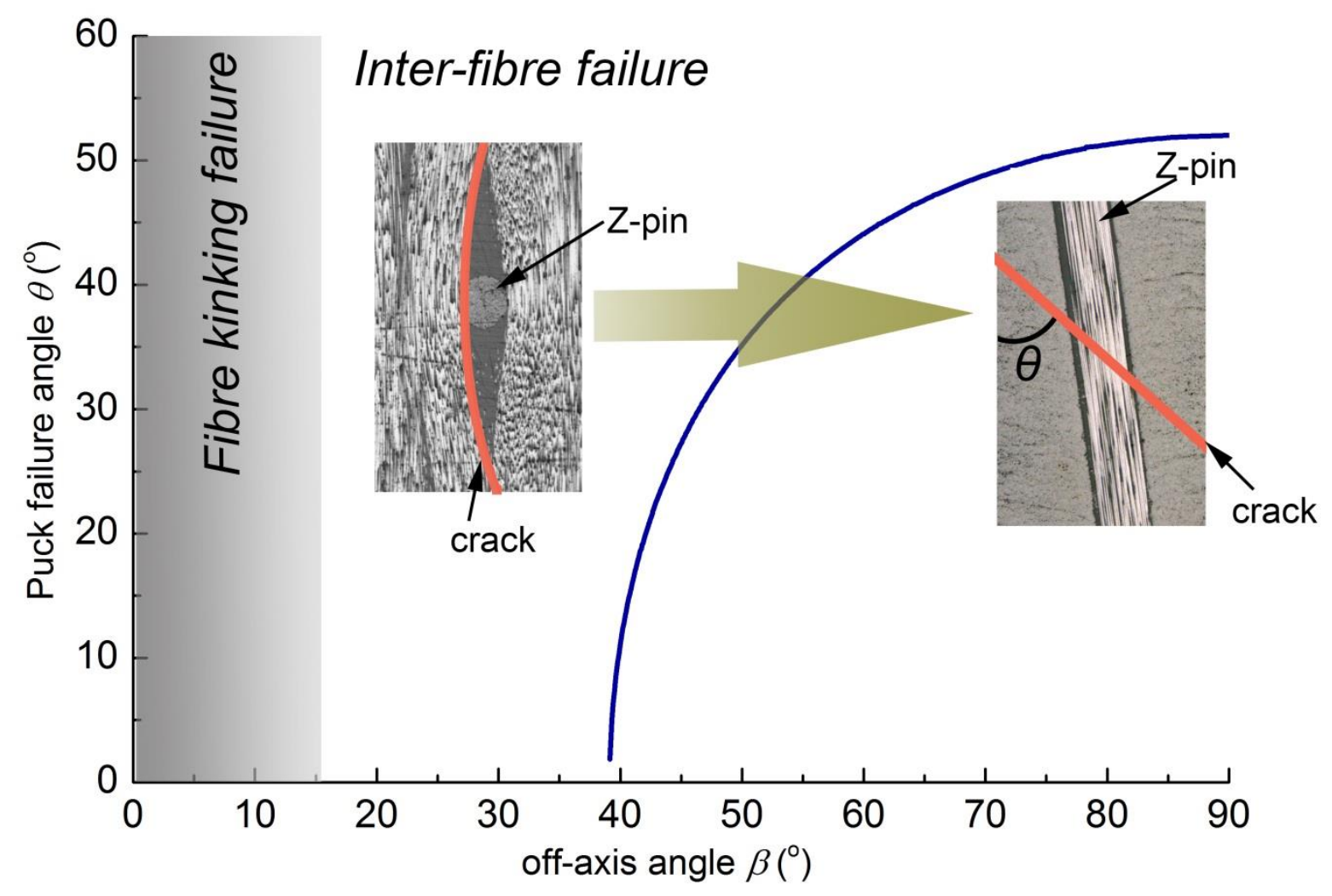

Fig.15 Change of Z-pin failure mode with compression at different off-axis angle

\section{Conclusions}

The influence of Z-pinning on the inter-fibre properties of unidirectional laminates was investigated in this study. The analysis of the Z-pinned laminates' microstructure revealed the distribution of fibre volume fraction in Z-pinned laminates, and it is found that the resin-rich zone was responsible for the decrease of fibre volume fraction in Z-pinned laminates. 
The modulus of laminates was reduced considerably with Z-pinning, and it was attributed to the decrease in fibre volume fraction as a result of the resin rich zones.

The failure strain and strength decreased with the presence of the Z-pins in shear dominated failure modes, while increased in the compression dominated failure modes. The change in the angle of the inter-fibre failure plane, resulting in different failure modes for Zpins and the resin-rich zone, was responsible for the influence of Z-pinning on the inter-fibre failure performance.

The influence of Z-pinning on the inter-fibre failure of unidirectional laminates was relatively moderate. The strength dropped by less than $10 \%$ for the $2 \% \mathrm{Z}$-pin volume fraction in the unidirectional laminates. Further studies on multi-directional laminates are recommended to be more representative for industrial applications.

The conclusion from this study is applicable to through-thickness reinforcement made with carbon fibre composites, such as T300/BMI used here. The bonding strength between pin and laminates may change with pin materials, and therefore affect the in-plane performance.

The failure of Z-pinned laminates under transverse tension load will be conducted in future, to generate a complete inter-fibre failure envelope for design guidance in industry.

\section{Acknowledgement}

The financial support from Engineering and Physical Sciences Research Council (EPSRC) of this research through grant No. EP/M012905/1 is gratefully acknowledged. 


\section{Reference}

[1] C. Canturri, E.S. Greenhalgh, S.T. Pinho, The relationship between mixed-mode II/III delamination and delamination migration in composite laminates, Composites Science and Technology 105 (2014) 102-109.

[2] D. Wang, L. Ye, Y. Tang, Y. Lu, Monitoring of delamination onset and growth during Mode I and Mode II interlaminar fracture tests using guided waves, Composites Science and Technology 72(2) (2012) 145-151.

[3] A.P. Mouritz, Review of z-pinned composite laminates, Composites Part A: Applied Science and Manufacturing 38(12) (2007) 2383-2397.

[4] S.-C. Dai, W. Yan, H.-Y. Liu, Y.-W. Mai, Experimental study on z-pin bridging law by pullout test, Composites Science and Technology 64(16) (2004) 2451-2457.

[5] D.D.R. Cartié, B.N. Cox, N.A. Fleck, Mechanisms of crack bridging by composite and metallic rods, Composites Part A: Applied Science and Manufacturing 35(11) (2004) $1325-1336$.

[6] K.L. Rugg, B.N. Cox, R. Massabo, Mixed mode delamination of polymer composite laminates reinforced through the thickness by Z-fibers, Composites Part A: Applied Science and Manufacturing 33 (2002).

[7] C.B.N. Rugg K. L, Sherrick G. O, Damage mechanisms for angled throughthickness rod reinforcement in carbon-epoxy laminates, Composites Part A: Applied Science and Manufacturing 29A (1998).

[8] D.D.R. Cartié, Effect of Z-Fibres on the delamination behaviour of carbon fibre / epoxy laminates, School of Industrial and Manufacturing Science, Cranfield University, 2000. 
[9] F. Pegorin, K. Pingkarawat, S. Daynes, A.P. Mouritz, Influence of z-pin length on the delamination fracture toughness and fatigue resistance of pinned composites, Composites Part B: Engineering 78 (2015) 298-307.

[10] F. Bianchi, X. Zhang, Predicting mode-II delamination suppression in z-pinned laminates, Composites Science and Technology 72(8) (2012) 924-932.

[11] F. Pegorin, K. Pingkarawat, A.P. Mouritz, Comparative study of the mode I and mode II delamination fatigue properties of z-pinned aircraft composites, Mater Design 65 (2015) 139-146.

[12] M.Z. Grassi, X; Meo, M, Prediction of stiffness and stresses in z-fibre reinforced composite laminates, Composites Part A: Applied Science and Manufacturing 33 (2002).

[13] T.M. Koh, S. Feih, A.P. Mouritz, Experimental determination of the structural properties and strengthening mechanisms of z-pinned composite T-joints, Composite Structures 93(9) (2011) 2222-2230.

[14] A.M. Nanayakkara, S. Feih, A.P. Mouritz, Improving the fracture resistance of sandwich composite T-joints by z-pinning, Composite Structures 96 (2013) 207-215.

[15] A. Knopp, G. Scharr, Effect of z-pin surface treatment on delamination and debonding properties of z-pinned composite laminates, Journal of Materials Science 49(4) (2013) 1674-1683.

[16] G. Allegri, X. Zhang, On the delamination and debond suppression in structural joints by Z-fibre pinning, Composites Part A: Applied Science and Manufacturing 38(4) (2007) 1107-1115. 
[17] C.A. Steeves, N.A. Fleck, In-plane properties of composite laminates with through-thickness pin reinforcement, International Journal of Solids and Structures 43(10) (2006) 3197-3212.

[18] P. Chang, A.P. Mouritz, B.N. Cox, Properties and failure mechanisms of zpinned laminates in monotonic and cyclic tension, Composites Part A: Applied Science and Manufacturing 37(10) (2006) 1501-1513.

[19] A.P. Mouritz, Compression properties of z-pinned composite laminates, Composites Science and Technology 67(15-16) (2007) 3110-3120.

[20] A. Knopp, C. Düsterhöft, M. Reichel, G. Scharr, Flexural properties of z-pinned composite laminates in seawater environment, Journal of Materials Science 49(24) (2014) 8343-8354.

[21] R.D. Sweeting, R.S. Thomson, The effect of thermal mismatch on Z-pinned laminated composite structures, Composite Structures 66(1-4) (2004) 189-195.

[22] H. Cui, Y. Li, S. Koussios, A. Beukers, Mixed mode cohesive law for Z-pinned composite analyses, Comp Mater Sci 75 (2013) 60-68.

[23] H. Koerber, J. Xavier, P.P. Camanho, High strain rate characterisation of unidirectional carbon-epoxy IM7-8552 in transverse compression and in-plane shear using digital image correlation, Mechanics of Materials 42(11) (2010) 1004-1019.

[24] D.M. Thomson, B. Erice, H. Cui, J. Hoffmann, J. Wiegand, N. Petrinic, A Puckbased localisation plane theory for rate- and pressure-dependent constitutive modelling of unidirectional fibre-reinforced polymers, Composite Structures 184 (2018) 299-305. 
[25] R. Maurin, C. Baley, D.D.R. Cartié, P. Davies, Influence of Through-Thickness Pinning on Composite Shear Properties, Appl Compos Mater 19(6) (2011) 853-864.

[26] H. Cui, M. Yasaee, G. Kalwak, A. Pellegrino, I.K. Partridge, S.R. Hallett, G. Allegri, N. Petrinic, Bridging mechanisms of through-thickness reinforcement in dynamic mode I\&II delamination, Composites Part A: Applied Science and Manufacturing 99 (2017) 198-207.

[27] A. Puck, H. Schurmann, Failure analysis of FRP laminates by means of physically based phenomenological models, Composites Science and Technology 58 (1998) 23.

[28] J. Wiegand, N. Petrinic, B. Elliott, An algorithm for determination of the fracture angle for the three-dimensional Puck matrix failure criterion for UD composites, Composites Science and Technology 68(12) (2008) 2511-2517.

[29] H. Cui, D. Thomson, A. Pellegrino, J. Wiegand, N. Petrinic, Effect of strain rate and fibre rotation on the in-plane shear response of $\pm 45^{\circ}$ laminates in tension and compression tests, Composites Science and Technology 135 (2016) 106-115.

[30] J. Wiegand, Constitutive modelling of composite materials under impact loading, Department of Engineering Science, University of Oxford, Oxford, 2009, p. 251.

[31] M. Yasaee, J.K. Lander, G. Allegri, S.R. Hallett, Experimental characterisation of mixed mode traction-displacement relationships for a single carbon composite Z-pin, Composites Science and Technology 94 (2014) 123-131. 


\title{
Inter-fibre failure of through-thickness reinforced laminates in combined transverse compression and shear load
}

\author{
Cui, Hao
}

\section{Elsevier}

Cui $\mathrm{H}$, Yasaee M, Melro A, Inter-fibre failure of through-thickness reinforced laminates in combined transverse compression and shear load, Composites Science and Technology, Volume 165, Issue September 2018, pp. 48-57

http://dx.doi.org/10.1016/j.compscitech.2018.06.011

Downloaded from Cranfield Library Services E-Repository 